\title{
Uniform Computation of the Error Function and Other Related Functions
}

\author{
By F. Matta and A. Reichel
}

Abstract. Uniform methods of computation, to any required degree of accuracy, for the error and other closely related functions are given.

1. Introduction. In a paper by Chiarella and Reichel [1], the function

$$
W_{0}(x, t)=\frac{w}{(4 \pi t)^{1 / 2}} \int_{-\infty}^{\infty} \frac{e^{-u^{2}} d u}{u^{2}+w^{2}}=\left(\frac{\pi}{4 t}\right)^{1 / 2} e^{w^{2}} \operatorname{erfc} w
$$

was expressed in the quadrature form

$$
W_{0}(x, t)=\frac{h}{w(4 \pi t)^{1 / 2}}+\frac{2 h w}{(4 \pi t)^{1 / 2}} \sum_{n=1}^{\infty} \frac{e^{-n^{2} h^{2}}}{n^{2} h^{2}+w^{2}}+\frac{\pi e^{w^{2}}}{(\pi t)^{1 / 2}\left(1-e^{2 \pi w / h}\right)}-\frac{w}{(4 \pi t)^{1 / 2}} E(h),
$$

where $w=(1-i x) / 2 t^{1 / 2}$, and the term involving $E(h)$ is a small error term. The net size $h$ was chosen sufficiently small so that the poles at $z= \pm i w$ of the integrand of

$$
\int_{c} \frac{e^{-z^{2}} d z}{\left(z^{2}+w^{2}\right)\left(1-e^{-2 \pi i z / h}\right)},
$$

from which the above quadrature formula was derived, were included in the contour $c$. This means that $t$ was restricted to the range $t>h^{2} / 4 \pi^{2}$.

In this paper, we extend the method to include the cases when $h$ is such that the poles at $z= \pm i w$ lie on and outside the contour $c$.

The respective results are

(1)

$$
\begin{aligned}
W_{0}(x, t)= & \frac{h}{w(4 \pi t)^{1 / 2}}+\frac{2 h w}{(4 \pi t)^{1 / 2}} \sum_{n=1}^{\infty} \frac{e^{-n^{2} h^{2}}}{n^{2} h^{2}+w^{2}} \\
& +P, \quad \text { if } t>h^{2} / 4 \pi^{2} \\
& +\frac{1}{2} P, \quad \text { if } t=h^{2} / 4 \pi^{2} \\
& +0, \quad \text { if } t<h^{2} / 4 \pi^{2} \\
& -\frac{h}{(4 \pi t)^{1 / 2}} E(h),
\end{aligned}
$$

where

$$
P=\frac{\pi e^{\omega^{2}}}{(\pi t)^{1 / 2}\left(1-e^{2 \pi \omega / h}\right)}
$$

Received September 2, 1969, revised September 28, 1970.

AMS 1970 subject classifications. Primary 33A20, 33A70; Secondary 33A20.

Key words and phrases. Voigt function, error function, Dawson's function, Fresnel integrals, rocket flight functions. 
2. Computation Formulae. The expressions for $W_{0}(x, t)$ given by (1) produce uniform methods for computing various related functions in mathematical physics. Formulae connecting $W_{0}(x, t)$ with such related functions have been given by Reichel [2].

(a) Voigt Functions. The function $W_{0}(x, t)$ can be expressed as

$$
W_{0}(x, t)=U_{0}(x, t)+i V_{0}(x, t),
$$

where

$$
\begin{aligned}
& U_{0}(x, t)=\frac{1}{(4 \pi t)^{1 / 2}} \int_{-\infty}^{\infty} \frac{e^{-(x-y)^{2} / 4 t}}{1+y^{2}} d y, \\
& V_{0}(x, t)=\frac{1}{(4 \pi t)^{1 / 2}} \int_{-\infty}^{\infty} \frac{e^{-(x-y)^{2 / 4 t}}}{1+y^{2}} y d y
\end{aligned}
$$

are Voigt functions. From (1), on separating real and imaginary parts, we obtain

$$
\begin{aligned}
U_{0}(x, t)= & \frac{h}{\sqrt{ } \pi\left(1+x^{2}\right)}+\frac{2 h}{\sqrt{ } \pi} \sum_{n=1}^{\infty} \frac{e^{-n^{2} h^{2}}\left(1+x^{2}+4 t n^{2} h^{2}\right)}{\left(1-x^{2}+4 n^{2} h^{2}\right)^{2}+4 x^{2}} \\
& +P_{1}, \quad \text { if } t>h^{2} / 4 \pi^{2} \\
& +\frac{1}{2} P_{1}, \quad \text { if } t=h^{2} / 4 \pi^{2} \\
& +0, \quad \text { if } t<h^{2} / 4 \pi^{2} \\
& -\frac{E(h)}{4 \sqrt{ } \pi t}, \\
V_{0}(x, t)= & \frac{h x}{\sqrt{ } \pi\left(1+x^{2}\right)}+\frac{2 h x}{\sqrt{ } \pi} \sum_{n=1}^{\infty} \frac{e^{-n^{2} h^{2}}\left(1+x^{2}-4 t n^{2} h^{2}\right)}{\left(1-x^{2}+4 t n^{2} h^{2}\right)^{2}+4 x^{2}} \\
& -Q_{1}, \quad \text { if } t>h^{2} / 4 \pi^{2}, \\
& -\frac{1}{2} Q_{1}, \quad \text { if } t=h^{2} / 4 \pi^{2}, \\
& -0, \quad \text { if } t<h^{2} / 4 \pi^{2}, \\
& +\frac{x E(h)}{4 \sqrt{ } \pi t},
\end{aligned}
$$

where

$$
\begin{array}{cc}
P_{1}=\left(\frac{\pi}{t}\right)^{1 / 2} e^{-\left(x^{2} / 4 t+\pi / h \sqrt{ } t-1 / 4 t\right)}\left[\frac{A C-B D}{C^{2}+D^{2}}\right], \\
Q_{1}=\left(\frac{\pi}{t}\right)^{1 / 2} e^{-\left(x^{2} / 4 t+\pi / h \sqrt{ } t-1 / 4 t\right)}\left[\frac{B C+A D}{C^{2}+D^{2}}\right], \\
A=\cos x / 2 t, \quad B=\sin x / 2 t, \\
C=e^{-\pi / h \sqrt{ } t}-\cos \pi x / h \sqrt{ } t, & D=\sin \pi x / h \sqrt{ } t .
\end{array}
$$

Suitable substitution and separation of terms in a formula given by Luke [3], [8] for the function erfc (az) yields the first two terms of Eqs. (2.1) and (2.2). However, the error term in Luke's formula is a combination of the last two terms of (2.1) and (2.2) and hence, in our context, depends on the variables $x$ and $t$. Formulae (2.1) and (2.2) require less computation than the method of Salzer [4] and the equivalent method given by Abramowitz and Stegun [5]. 
Many workers in optics and astrophysics use a Voigt function in the form

$$
H(a, u)=\frac{a}{\pi} \int_{-\infty}^{\infty} \frac{e^{-y^{2}} d y}{(u-y)^{2}+a^{2}}=(1 / a \sqrt{ } \pi) U_{0}\left(u / a, 1 / 4 a^{2}\right)
$$

so that

$$
\begin{aligned}
H(a, u)= & \frac{h a}{\pi\left(a^{2}+u^{2}\right)}+\frac{2 a h}{\pi} \sum_{n=1}^{\infty} \frac{e^{-n^{2} h^{2}}\left(a^{2}+u^{2}+n^{2} h^{2}\right)}{\left(a^{2}-u^{2}+n^{2} h^{2}\right)^{2}+4 a^{2} u^{2}} \\
& +P_{2}, \quad \text { if } a<\pi / h, \\
& +\frac{1}{2} P_{2}, \quad \text { if } a=\pi / h, \\
& +0, \quad \text { if } a>\pi / h, \\
& -\frac{a}{\pi} E(h) .
\end{aligned}
$$

Similarly, if $K(a, u)=(1 / a \sqrt{ } \pi) V_{0}\left(u / a, 1 / 4 a^{2}\right)$ then

$$
\begin{aligned}
K(a, u)= & \frac{h u}{\pi\left(a^{2}+u^{2}\right)}+\frac{2 u h}{\pi} \sum_{n=1}^{\infty} \frac{e^{-n^{2} h^{2}}\left(a^{2}+u^{2}-n^{2} h^{2}\right)}{\left(a^{2}-u^{2}+n^{2} h^{2}\right)^{2}+4 a^{2} u^{2}} \\
& -Q_{2}, \quad \text { if } a<\pi / h \\
& -\frac{1}{2} Q_{2}, \quad \text { if } a=\pi / h, \\
& -0, \quad \text { if } a>\pi / h \\
& +\frac{u E(h)}{\pi},
\end{aligned}
$$

where

$$
\begin{gathered}
P_{2}=2 e^{-\left(u^{2}+2 a \pi / h-a^{2}\right)}\left[\frac{A_{1} C_{1}-B_{1} D_{1}}{C_{1}^{2}+D_{1}^{2}}\right], \\
Q_{2}=2 e^{-\left(u^{3}+2 a \pi / h-a^{2}\right)}\left[\frac{A_{1} D_{1}+B_{1} C_{1}}{C_{1}^{2}+D_{1}^{2}}\right], \\
A_{1}=\cos 2 a u, \quad B_{1}=\sin 2 a u, \\
C_{1}=e^{-2 a \pi / h}-\cos 2 u \pi / h, \quad D_{1}=\sin 2 u \pi / h .
\end{gathered}
$$

(b) Error Function of Complex Argument. The function

$$
\operatorname{erfc} z=\frac{2}{\sqrt{ } \pi} \int_{z}^{\infty} e^{-u^{2}} d u=\alpha-i \beta,
$$

where $z=x+i y$, can be computed from

$$
\begin{aligned}
& \alpha=e^{y^{2}-x^{2}}[\cos 2 x y H(x, y)-\sin 2 x y K(x, y)], \\
& \beta=e^{y^{2}-x^{2}}[\sin 2 x y H(x, y)+\cos 2 x y K(x, y)] .
\end{aligned}
$$

Some workers use the function

$$
\begin{aligned}
w(z)=e^{-z^{2}} \operatorname{erfc}(-i z) & =\frac{i}{\pi} \int_{-\infty}^{\infty} \frac{e^{-t^{2}} d t}{z-t}, & & \operatorname{Im} z>0, \\
& =\frac{i}{\pi} \int_{-\infty}^{\infty} \frac{e^{-t^{2}} d t}{z-t}+2 e^{-z^{2}}, & & \operatorname{Im} z<0,
\end{aligned}
$$


which can be computed from

$$
w(z)=H(y, x)+i K(y, x) .
$$

A useful survey regarding the properties of this function, and computational methods, may be found in [3], [6], [7] and [8]. In references [3] and [8], Luke gives expressions for the complex error function in terms of the modified Bessel function of the second kind. For computational purposes, the expressions presented in the present paper require less computing time.

(c) Dawson's Function of Complex Argument. The function

$$
w(z)=e^{-z^{2}} \int_{0}^{z} e^{u^{z}} d u=u(x, y)+i v(x, y)
$$

may be computed from

$$
\begin{aligned}
& u(x, y)=\frac{\sqrt{ } \pi}{2}\left(\sin 2 x y e^{\nu^{2}-x^{3}}+K(y, x)\right), \\
& v(x, y)=\frac{\sqrt{ } \pi}{2}\left(\cos 2 x y e^{y^{2}-x^{2}}-H(y, x)\right) .
\end{aligned}
$$

(d) Error Function of Real Argument.

$$
\begin{aligned}
\operatorname{erfc} x= & \frac{2}{\sqrt{ } \pi} \int_{x}^{\infty} e^{-u^{2}} d u \\
= & \frac{h e^{-x^{2}}}{x \pi}\left[1+2 x^{2} \sum_{n=1}^{\infty} \frac{e^{-n^{2} h^{2}}}{n^{2} h^{2}+x^{2}}\right] \\
& -2 /\left(e^{2 \pi x / h}-1\right), \quad \text { if } x<\pi / h, \\
& -1 /\left(e^{2 \pi x / h}-1\right), \quad \text { if } x=\pi / h, \\
& -0, \quad \text { if } x>\pi / h, \\
& -x e^{-x^{2}} E(h) / \pi .
\end{aligned}
$$

(e) Dawson's Function of Real Argument.

$$
\begin{aligned}
w(x)= & e^{-x^{3}} \int_{0}^{x} e^{u^{2}} d u \\
= & \frac{h}{2 \sqrt{ } \pi x}-\frac{x h}{\sqrt{ } \pi} \sum_{n=1}^{\infty} \frac{e^{-n^{2} h^{2}}}{n^{2} h^{2}-x^{2}} \\
& -\frac{\sqrt{ } \pi}{2} e^{-x^{2}} \cot \pi x / h, \quad \text { if } x<\pi / h, \\
& -\frac{\sqrt{ } \pi}{4} e^{-x^{2}} \cot \pi x / h, \quad \text { if } x=\pi / h, \\
& -0, \quad \text { if } x>\pi / h, \\
& +\frac{x E(h)}{2 \sqrt{ } \pi} .
\end{aligned}
$$


Note that with an error of smaller order than $E(h)$, we can write

$$
w(x)=\frac{h x}{2 \sqrt{ } \pi} \sum_{n=-\infty}^{\infty} \frac{e^{-x^{2}}-e^{-n^{2} h^{2}}}{n^{2} h^{2}-x^{2}} .
$$

(f) The Fresnel Integrals. The integrals

$$
c(x)=\sqrt{\frac{2}{\pi}} \int_{0}^{\sqrt{ } x} \cos t^{2} d t, \quad s(x)=\sqrt{\frac{2}{\pi}} \int_{0}^{\sqrt{ } x} \sin t^{2} d t
$$

may be computed from

$$
\begin{aligned}
c(x)=\frac{1}{2}-\frac{1}{2 \sqrt{ }(\pi x)}\{\cos x & {\left[H\left(\sqrt{\frac{x}{2}}, \sqrt{\frac{x}{2}}\right)-K\left(\sqrt{\frac{x}{2}}, \sqrt{\frac{x}{2}}\right)\right] } \\
& \left.-\sin x\left[H\left(\sqrt{\frac{x}{2}}, \sqrt{\frac{x}{2}}\right)+K\left(\sqrt{\frac{x}{2}}, \sqrt{\frac{x}{2}}\right)\right]\right\}, \\
s(x)=\frac{1}{2}-\frac{1}{2 \sqrt{ }(\pi x)}\{\sin x & {\left[H\left(\sqrt{\frac{x}{2}}, \sqrt{\frac{x}{2}}\right)-K\left(\sqrt{\frac{x}{2}}, \sqrt{\frac{x}{2}}\right)\right] } \\
& \left.+\cos x\left[H\left(\sqrt{\frac{x}{2}}, \sqrt{\frac{x}{2}}\right)+K\left(\sqrt{\frac{x}{2}}, \sqrt{\frac{x}{2}}\right)\right]\right\} .
\end{aligned}
$$

(g) The Rocket Flight Functions. The functions

$$
\begin{aligned}
& A(x)=\frac{1}{\pi \sqrt{ } 2} \int_{0}^{\infty} \frac{e^{-\pi x^{2} u / 2} u^{-1 / 2} d u}{1+u^{2}}, \\
& B(x)=\frac{1}{\pi \sqrt{ } 2} \int_{0}^{\infty} \frac{e^{-\pi x^{2} u / 2} u^{1 / 2} d u}{1+u^{2}}
\end{aligned}
$$

can be computed from

$$
\begin{aligned}
& A(x)=\frac{1}{2}\left[H\left(\frac{x \sqrt{ } \pi}{2}, \frac{x \sqrt{ } \pi}{2}\right)+K\left(\frac{x \sqrt{ } \pi}{2}, \frac{x \sqrt{ } \pi}{2}\right)\right], \\
& B(x)=\frac{1}{2}\left[H\left(\frac{x \sqrt{ } \pi}{2}, \frac{x \sqrt{ } \pi}{2}\right)-K\left(\frac{x \sqrt{ } \pi}{2}, \frac{x \sqrt{ } \pi}{2}\right)\right] .
\end{aligned}
$$

3. Hints on Computation. (i) The authors [1] note that

$$
|E(h)| \leqq \frac{2 \sqrt{ } \pi e^{-\pi^{2} / h^{2}}}{1-e^{-\pi^{2} / h^{2}}},
$$

which gives the following bounds on $E(h)$ :

\begin{tabular}{cccccc}
\hline$h$ & 1 & 0.8 & 0.75 & 0.6 & 0.5 \\
\hline$E(h)$ & $10^{-4}$ & $10^{-6}$ & $10^{-7}$ & $10^{-11}$ & $10^{-15}$ \\
\hline
\end{tabular}

If we take $h=\frac{1}{2}$, the term containing $E(h)$ in all the formulae may be neglected, with an error of the order $10^{-15}$, for a large range of arguments.

(ii) With $h=\frac{1}{2}$, the term $\frac{1}{2} P$ in Eq. (1), corresponding to the case when the poles at $z= \pm i w$ lie on the contour $c$, can be neglected with an error less than $10^{-15}$. 
Similarly, the term obtained from this $\frac{1}{2} P$ in each of the given expressions can be neglected with an error of $10^{-15}$.

(iii) Note also that the term $P$ in Eq. (1) and the corresponding term in each of the other formulae can be neglected with an error less than $10^{-15}$, if the exponent of the exponential is less than -34 .

(iv) In all formulae, except (2.11), about 12 terms are required in the summation to obtain accuracy of order $10^{-15}$. The coefficients $e^{-n^{2} h^{2}}$ and $n^{2} h^{2}$ can be computed and entered before the computation, for a given number of arguments, begins. To obtain more accuracy, $h$ can be decreased and the number of terms in the summation increased.

(v) The only function for which the given expression is difficult to compute as it stands is Dawson's function of real argument, Eqs. (2.10) and (2.11). In Eq. (2.10), we chose $h=.55$ and computed $w(x)$ for all $x$ except when $\left|n^{2} h^{2}-x^{2}\right|<0.1$. For such values of $x$ we chose $h=.45$, which avoided the zero in the denominator of the summation term. Of the two formulae (2.10) and (2.11), the first requires less computation.

Department of Applied Mathematics

The University of Sydney

Sydney, Australia

1. C. Chiarella \& A. Reichel, "On the evaluation of integrals related to the error function," Math. Comp., v. 22, 1968, pp. 137-143. MR 36 \#6117.

2. A. REICHEL, J. Quant. Spectrosc. Radiat. Transfer, v. 8, 1968, p. 1601. \#5198.

3. Y. L. LuKE, Integrals of Bessel Functions, McGraw-Hill, New York, 1962. MR 25

4. H. E. SALzER, "Formulas for calculating the error function of a complex variable," $M T A C$, v. 5, 1951, pp. 67-70. MR 13, 989; 1140.

5. M. ABramowitz \& I. A. STEgUN (Editors), Handbook of Mathematical Functions, with Formulas, Graphs and Mathematical Tables, Nat. Bur. Standards Appl. Math. Series, 55, Superintendent of Documents, U. S. Government Printing Office, Washington, D. C., 1964. MR 29 \#4914.

6. B. H. ARmstrong, J. Quant. Spectrosc. Radiat. Transfer, v. 7, 1967, p. 61.

7. H. C. THACHER, Computation of the Complex Error Function by Continued Fractions, Blanche Anniversary Volume, Aerospace Res. Lab., U. S. Air Force, Washington, D. C., 1967, pp. 315-337. MR 35 \#2464.

8. Y. L. LUKE, The Special Functions and Their Approximations. Vol. 2, Math. in Sci. and Engrg., vol. 53, Academic Press, New York, 1969. MR 40 \#2909. 